\title{
Erratum to: Convergence to fractional kinetics for random walks associated with unbounded conductances
}

\author{
Martin T. Barlow • Jiří Černý
}

Published online: 11 February 2011

(C) Springer-Verlag 2011

\section{Erratum to: Probab. Theory Relat. Fields DOI 10.1007/s00440-009-0257-z}

The paper [1] contains two rather important mistakes; we are grateful to the anonymous referee of the follow-up paper [3] for pointing them out.

The first mistake concerns the topology used in two main theorems of the paper. Due to measurability issues, see [2, Chap. 18], it is not possible to define the distribution of the processes $X_{n}$ on the Skorokhod space endowed with the uniform topology, and therefore the usual $J_{1}$-topology should be used. The correct statement of Theorem 1.2 follows. Theorem 1.3 should be corrected accordingly.

Theorem 1.2 Assume that $d \geq 3$,

$$
\mathbb{P}\left[\mu_{e} \geq u\right]=C_{1} u^{-\alpha}(1+o(1)), \quad u \rightarrow \infty,
$$

for some $\alpha \in(0,1), C_{1} \in(0, \infty)$, and that $\mathbb{P}\left[\mu_{e}>\underline{c}\right]=1$ for some $\underline{c} \in(0, \infty)$. Let

$$
X_{n}(t)=n^{-1} X\left(t n^{2 / \alpha}\right), \quad t \in[0, \infty), n \in \mathbb{N},
$$

The online version of the original article can be found under doi:10.1007/s00440-009-0257-z.

\section{T. Barlow}

Department of Mathematics, University of British Columbia, Vancouver, BC, V6T 1Z2, Canada e-mail: barlow@math.ubc.ca

J. Černý $(\bowtie)$

Department of Mathematics, ETHZ Zürich, Rämistr. 101, 8092 Zürich, Switzerland e-mail: jiri.cerny@math.ethz.ch 
be the rescaled CSRW. Then there exists a constant $\mathcal{C} \in(0, \infty)$ such that $\mathbb{P}$-a.s., under $P_{0}^{\mu}$, the sequence of processes $X_{n}$ converges in law to a multiple of the fractionalkinetics process $\mathcal{C} \mathrm{FK}_{d, \alpha}$ on $D^{d}$ equipped with the Skorokhod $J_{1}$-topology.

The change of the topology does not influence the proof of the theorem appearing at the end of Sect. 8 (ignoring the obvious replacements of the uniform by the $J_{1}$-topology). A suitable continuity of the composition map in the $J_{1}$-topology is proved in [4, Theorem 13.2.2].

The second mistake appears in the coarse-graining construction of Sect. 6. The proof of Proposition 6.7 is not complete and in fact the result may not be true. Therefore it is necessary to weaken the statement of Proposition 6.7 as follows.

Proposition 6.7 Let $T, \varepsilon_{s}, \varepsilon_{g}>0$. Define $s_{n}(i)=s_{n}\left(i, \varepsilon_{s}, \varepsilon_{s}, \varepsilon_{s}^{-1}, \varepsilon_{s}^{-1}\right)$ and let $\mathcal{F}_{i}^{n}$ be the $\sigma$-algebra generated by $\left(Y_{s}, s \leq t_{n}(i)\right)$. Then, there is a constant $\kappa>0$ independent of $\varepsilon_{s}, \varepsilon_{g}$ and $T$, such that $\mathbb{P}$-a.s. for all $n$ large enough, for all $i \leq \varepsilon_{g}^{-2} T$, and for all intervals $A=(0, a]$ with $a \in(0, \infty)$

$$
\begin{aligned}
& \left|P_{0}^{\mu}\left[s_{n}(i)=0 \mid \mathcal{F}_{i}^{n}\right]-\left(1-c_{\varepsilon_{s}} \varepsilon_{g}^{2}\right)\right| \leq \kappa c_{\varepsilon_{s}}^{2} \varepsilon_{g}^{4}, \\
& \left|P_{0}^{\mu}\left[s_{n}(i) \in A \mid \mathcal{F}_{i}^{n}\right]-\varepsilon_{g}^{2} v_{\varepsilon_{s}}(A)\right| \leq \kappa v_{\varepsilon_{s}}(A) c_{\varepsilon_{s}} \varepsilon_{g}^{4},
\end{aligned}
$$

where

$$
c_{\varepsilon_{s}}=\mathcal{C}_{Y}^{-2} \int_{\varepsilon_{s}}^{\varepsilon_{s}^{-1}} \int_{\varepsilon_{s}}^{\varepsilon_{s}^{-1}} \alpha v u^{-\alpha-1} \mathrm{~d} u F_{C}(\mathrm{~d} v)
$$

and $v_{\varepsilon_{s}}$ is the measure on $(0, \infty)$ given by

$$
v_{\varepsilon_{s}}(\mathrm{~d} x)=\mathcal{C}_{Y}^{-2} \int_{\varepsilon_{s}}^{\varepsilon_{s}^{-1}} \int_{\varepsilon_{s}}^{\varepsilon_{s}^{-1}} \frac{v}{2 u} \exp \left\{-\frac{x v}{2 u}\right\} \alpha v u^{-\alpha-1} \mathrm{~d} u F_{C}(\mathrm{~d} v) \mathrm{d} x .
$$

The proof of this proposition is then as in the original paper. The error terms of (1), (2) not appearing there are explained as follows. With one exception, all the errors in the proof of Proposition 6.7 can be made arbitrarily small with respect to $\varepsilon_{g}^{2}$ by choosing $\varepsilon_{o}, \varepsilon_{m}, \varepsilon_{s}$ small and $n$ large. The only exception is the error coming from the estimate on the probability that more than two edges from $E_{n}\left(\varepsilon_{s}\right)$ are visited. This probability can be bounded by the right-hand side of (1) as follows from the paragraph after (6.45). The error term in (2) then corresponds to the event that the first visited edge of $E_{n}\left(\varepsilon_{s}\right)$ gives (after the normalisation) a contribution belonging to $A=(0, a]$, and then another edge from $E_{n}\left(\varepsilon_{s}\right)$ is visited.

Proposition 6.7 is used on two places in the original paper. First, in the proof of Lemma 6.8 which should be restated as follows. 
Lemma 6.8 For all $\varepsilon_{g}, \varepsilon_{s}>0, \ell \in \mathbb{N}, \lambda_{1}, \ldots, \lambda_{\ell}>0, \xi_{1}, \ldots, \xi_{\ell} \in \mathbb{R}^{n}$, and $i_{1}<$ $\cdots<i_{\ell} \leq T \varepsilon_{g}^{-2}, \mathbb{P}$-a.s., for all $n$ large enough

$$
\begin{aligned}
& E_{0}^{\mu}\left[\exp \left\{-\sum_{j=1}^{\ell}\left[\lambda_{j} s_{n}\left(i_{j}\right)+\xi_{j} \cdot r_{n}\left(i_{j}\right)\right]\right\}\right] \\
& =\prod_{j=1}^{\ell}\left[1+\varepsilon_{g}^{2}\left(\frac{\left|\xi_{j}\right|^{2}}{2 d}-c_{\varepsilon_{s}}+G\left(\lambda_{j}\right)\right)\right]+\mathcal{R},
\end{aligned}
$$

where

$$
G(\lambda)=G^{\varepsilon_{s}}(\lambda)=\int_{0}^{\infty} e^{-\lambda x} v_{\varepsilon_{s}}(\mathrm{~d} x)=\mathcal{C}_{Y}^{-2} \int_{\varepsilon_{s}}^{\varepsilon_{s}^{-1}} \int_{\varepsilon_{s}}^{\varepsilon_{s}^{-1}} \frac{\alpha v^{2} u^{-\alpha-1}}{v+2 u \lambda} \mathrm{d} u F_{C}(\mathrm{~d} v) .
$$

and the reminder term $\mathcal{R}$ satisfies for all small $\varepsilon_{g}$ and finite constants $c\left(\xi_{j}, \lambda_{j}\right)$

$$
|\mathcal{R}| \leq \varepsilon_{g}^{3}\left(1+c_{\varepsilon_{s}}^{2}\right) \sum_{j=1}^{\ell} c\left(\xi_{j}, \lambda_{j}\right) .
$$

The straightforward modifications that are necessary to prove this lemma from the modified Proposition 6.7 are given in [3, Lemma 4.8].

The second application of Proposition 6.7 appears under (8.3) in the proof of Theorem 2.1. The proposition is used there to prove that the process $\sum_{i=1}^{\left.L \cdot \varepsilon_{g}^{-2}\right\rfloor} s_{n}(i)$ converges as $n \rightarrow \infty$ and $\varepsilon_{g} \rightarrow 0$ to a compound Poisson process with intensity measure $v_{\varepsilon_{s}}$. This statement however follows directly from the corrected Lemma 6.8 and the computation appearing in (8.7), (8.8): It is sufficient to set $\xi=0$ and observe that $c_{\varepsilon_{s}}-G(\lambda)=\int_{0}^{\infty}\left(1-e^{-\lambda x}\right) v_{\varepsilon_{s}}(\mathrm{~d} x)$ which is the Laplace exponent of such compound Poisson process.

\section{References}

1. Barlow, M.T., Černý, J.: Convergence to fractional kinetics for random walks associated with unbounded conductances, Probab. Theory Relat. Fields. Online first http://dx.doi.org/10.1007/s00440-009-0257-z

2. Billingsley, P.: Convergence of Probability Measures. Wiley, New York (1968)

3. Černý, J.: On two-dimensional random walk among heavy-tailed conductances. Electron. J. Probab. 16, 293-313 (2011)

4. Whitt, W.: Stochastic-process limits. In: Springer Series in Operations Research. Springer, New York (2002) 